\title{
Online Appendix for Evolutionary Selection of Individual Expectations and Aggregate Outcomes in Asset Pricing Experiments
}

\author{
By Mikhail Anufriev and Cars Hommes*
}

This Appendix consists of five sections. In Section A we illustrate aggregate price dynamics and individual forecasting behavior in all the sessions of the HSTV05 and HSTV08 experiments not covered in the main part of the paper. Section B collects a few results about existence and stability of the steady states for dynamics of the models with homogeneous expectations determined by the heuristics constituting the HSM. In Section C we return to the HSTV05 experiment and provide an evidence of heterogeneity within sessions and between sessions on the basis in terms of stability analysis of Section B. Finally, Section D complements Section 4 of the main paper by providing the fitting results of the HSM for all the sessions of the HSTV05 and HSTV08 experiments not covered in the main part of the paper.

\section{A. Price and Prediction Dynamics in the Experiments}

This Appendix provides a detailed view on the individual and aggregate dynamics in the HSTV05 and HSTV08 experiments. Fig. 2 of the main text provides an illustration of observed aggregate dynamics of price in all 20 sessions. Fig. 3 of the main text displays the detailed dynamics in 6 out of 20 sessions. The dynamics of the remaining 14 sessions is shown in Figs. A1-A3. For every panel an upper part shows prices in the experiment (line) in comparison with the fundamental level (dotted line). A lower part shows individual predictions of 6 participants with forecasting errors in an inner frame.

The left panels of Fig. A1 show sessions 2, 1 and 7 of the HSTV05 experiment with high fundamental price and robots. The patterns of monotone convergence, constant oscillations and dampened oscillations are similar with the corresponding patterns in sessions 5, 6 and 4 (cf. the left panels of Fig. 3 of the main text). The top right panel shows session 3 of the HSTV05 experiment, where one of the participants presumably made a typing error. Two remaining right panels show sessions 9 and 10 from the HSTV05 experiment with robots and low fundamental price.

* Anufriev: Economics Discipline Group, University of Technology Sydney, PO Box 123 Broadway, NSW 2007, Australia (e-mail: Mikhail.Anufriev@uts.edu.au). Hommes: CeNDEF, School of Economics, University of Amsterdam, Roetersstraat 11, NL-1018 WB Amsterdam, Netherlands (e-mail: C.H.Hommes@uva.nl). 

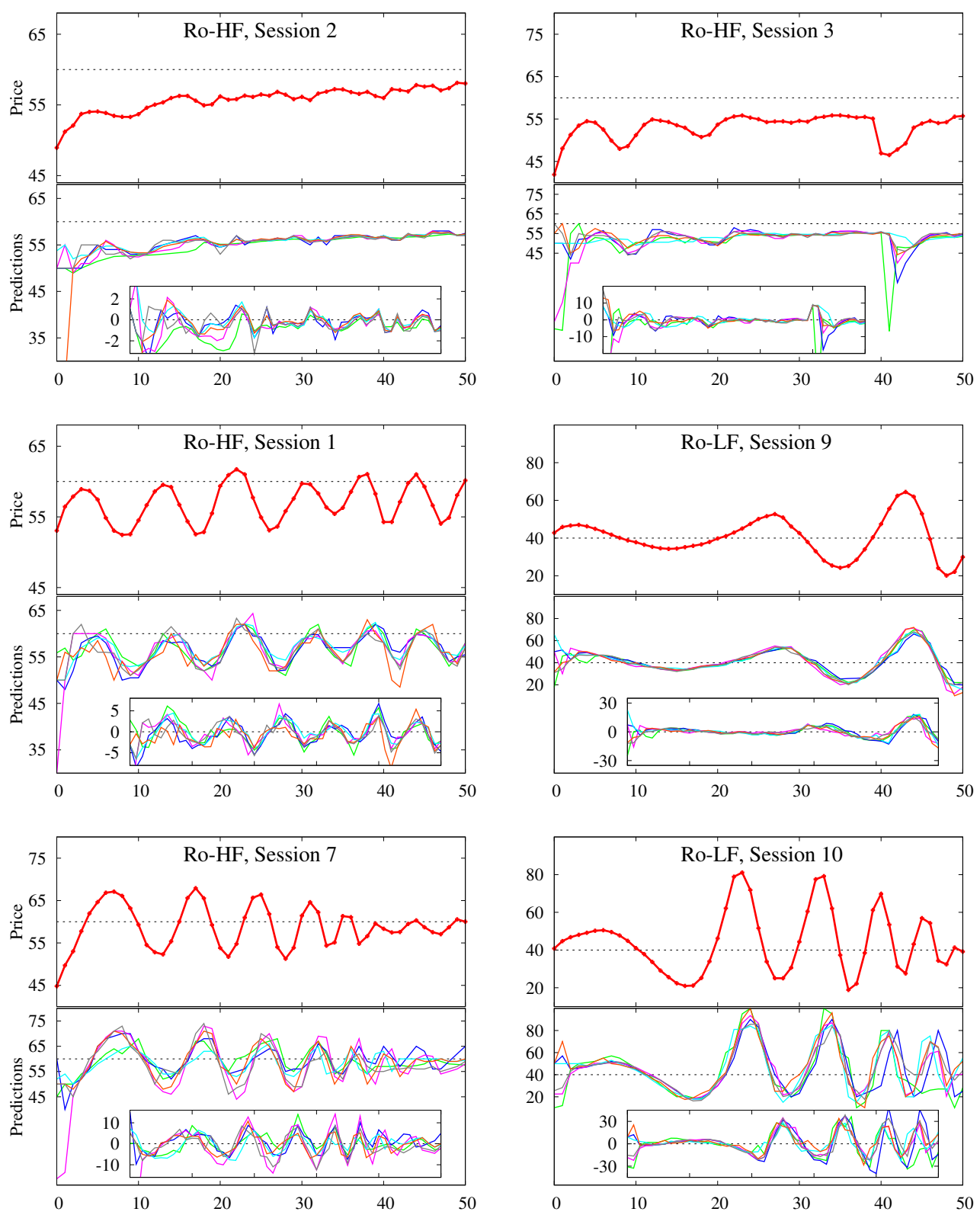

Figure A1. Prices, predictions and forecasting errors in 6 Sessions of the HSTV05 Experiment. In SESSiOns 2, 1, 7 AND 3 The FUndAMENTAL PRICE $p^{f}=60$, In SESSIONS 9 AND 10 The Fundamental PRICE $p^{f}=40$. 
Fig. A2 shows sessions 11, 13 and 14 of the HSTV05 experiment with high fundamental price $p^{f}=60$ without "robots".
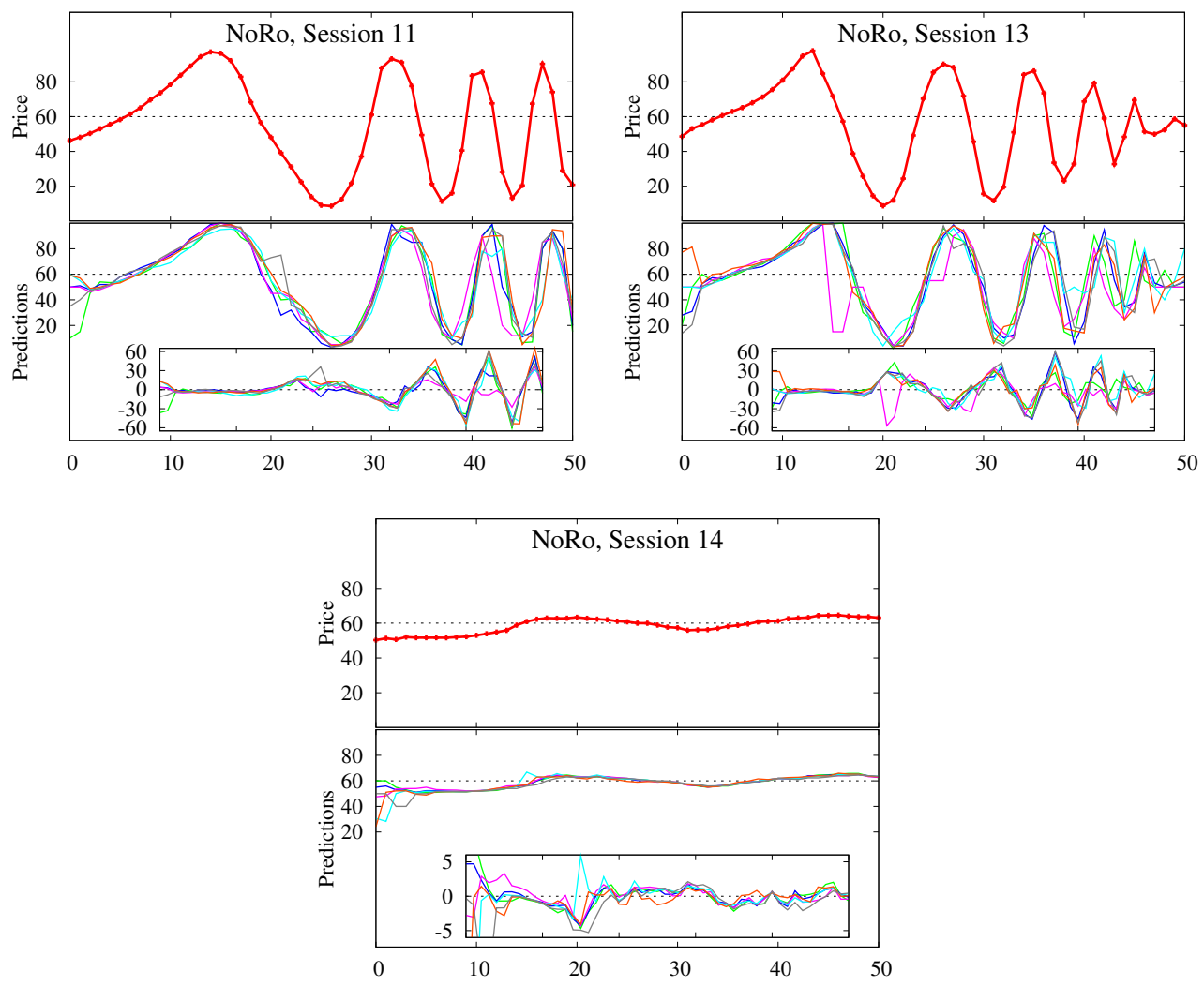

Figure A2. Prices, Predictions And forechsting ERrors in 3 SESsions of the HSTV05 Experiment Without ROBOts.

Fig. A3 shows sessions 1 and $3-6$ of the HSTV08 experiment with large forecasting range [0,1000]. In all the sessions except session 1 the large price bubble has been observed. 

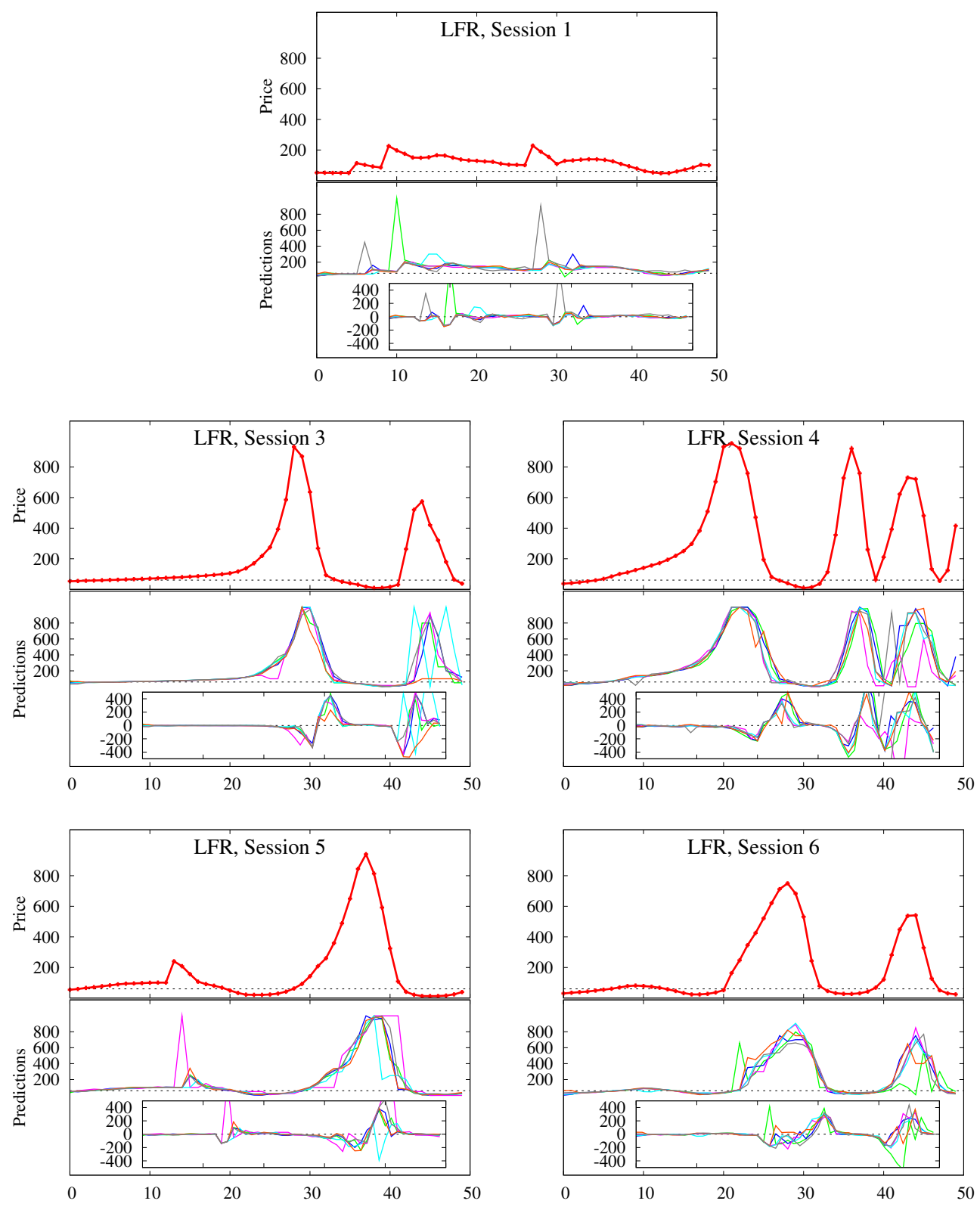

Figure A3. Prices, predictions and forecasting errors in 3 Sessions of the HSTV08 experiment With large forecasting Range. OCCASional "Spoilers" are Clearly Visible. 


\section{B. Homogeneous Expectations Model}

The model with homogeneous expectations is given by

$$
\left\{\begin{aligned}
p_{t+1}^{e} & =f\left(p_{t-1}, p_{t-2}, p_{t}^{e}\right) \\
n_{t} & =1-\exp \left(-\frac{1}{200}\left|p_{t-1}-p^{f}\right|\right) \\
p_{t} & =\frac{1}{1+r}\left(\left(1-n_{t}\right) p_{t+1}^{e}+n_{t} p^{f}+\bar{y}+\varepsilon_{t}\right) .
\end{aligned}\right.
$$

for the experiments with robots. The first equation describes forecasting behavior with a simple first order heuristic $f$ as in Eq. (5) of the main text, which can be either adaptive expectations (in which case $f$ does not depend on $p_{t-2}$ ) or trend following expectations (in which case $f$ does not depend on $p_{t}^{e}$ ). Two other equations give the evolution of the share of "robot" traders and prices, identical to the equations used in the experiment, cf. Eqs. (3) and (4) of the main text. When the robots are not present, the second equation should be omitted and Eq. (1) of the main text should be used in place of the third.

We present below an analysis of the so-called deterministic skeleton model, setting term $\varepsilon_{t}$ in (B1) to zero. Stochastic simulations with the same realizations of the shocks, $\varepsilon_{t}$, as in the experiment are given in Fig. 4 of the main text, in order to illustrate how the noise affects price fluctuations. In terms of deviations from the fundamental price the model can be rewritten as

$$
p_{t}-p^{f}=\frac{1}{1+r}\left(\left(1-n_{t}\right) p_{t+1}^{e}+n_{t} p^{f}-p^{f}\right)=\frac{1-n_{t}}{1+r}\left(p_{t+1}^{e}-p^{f}\right),
$$

Adaptive Expectations. - Under adaptive expectations $f$ is given by Eq. (6) of the main text. The following result describes the behavior of system (B1) in this case.

PROPOSITION 1: Consider the deterministic skeleton of system (B1) with the adaptive prediction rule given by Eq. (6) of the main text. This system has a unique steady-state with price equal to fundamental price, i.e., $p^{*}=p^{f}$. The steady-state is globally stable for $0<w \leq 1$, with a real eigenvalue $\lambda, 0<\lambda<1$, so that the convergence is monotonic.

\section{PROOF:}

From the general relation (B2) it follows that

$$
p_{t+1}^{e}-p^{f}=w\left(p_{t-1}-p^{f}\right)+(1-w)\left(p_{t}^{e}-p^{f}\right)=\left(p_{t}^{e}-p^{f}\right)\left(w \frac{1-n_{t-1}}{1+r}+1-w\right) .
$$

The expression in the last parenthesis is a convex combination of 1 and (1 $\left.n_{t-1}\right) /(1+r)<1$. For positive weight $w$ such combination is always less than 
1. Therefore, the dynamical system defines a contraction of expectations, which then must globally converge to $p^{f}$. The price realization in this point is uniquely defined from Eq. (6) of the main text as $p^{*}=p^{f}$. Finally, the evolution of robot traders implies that $n^{*}=0$ in this fixed-point.

Extrapolative Expectations. - Consider now the dynamics of the homogeneous expectations model with a rule given by

$$
p_{t+1}^{e}=\alpha+\beta_{1} p_{t-1}+\beta_{2} p_{t-2} .
$$

This extrapolative rule incorporates both the trend following and the anchor and adjustment heuristic as special cases. Indeed, setting $\alpha=0, \beta_{1}=1+\gamma$ and $\beta_{2}=-\gamma$, the trend-following heuristics given by Eq. (7) from the main text is obtained, while $\alpha=p^{f} / 2, \beta_{1}=1.5$ and $\beta_{2}=-1$ correspond to the anchoring and adjustment heuristic given by Eq. (8) from the main text.

The rules for which the forecasts are not consistent with realizations will be disregarded by the participants, sooner or later. Therefore, we confine our attention to the rules satisfying the following simple steady-state consistency requirement:

DEFINITION 1: The extrapolative rule (B3) is called consistent in the steadystate $p^{*}$, if it predicts $p^{*}$ in this steady-state.

In other words, consistent rules give unbiased predictions at the steady-state. Obviously, the extrapolative rule is consistent in $p^{*}$ if and only if $\alpha=\left(1-\beta_{1}-\right.$ $\left.\beta_{2}\right) p^{*}$. Notice that, the trend-following heuristic (Eq. (7) from the main text) is consistent at any steady-state, while the anchoring and adjustment heuristic (Eq. (8) from the main text) is consistent only at the steady state with $p^{*}=p^{f}$.

The following result describes all possible steady-states of the asset-pricing dynamics with consistent extrapolative heuristic, as well as their local stability.

PROPOSITION 2: Consider the dynamics of the deterministic skeleton of (B1) with extrapolative prediction rule (B3).

There exists a unique steady-state in which the rule is consistent. In this steadystate, $p^{*}=p^{f}$ and the fraction of robot traders $n^{*}=0$. The "fundamental" steady-state is locally stable if the following three conditions are met

$$
\beta_{2}<(1+r)-\beta_{1}, \quad \beta_{2}<(1+r)+\beta_{1}, \quad \beta_{2}>-(1+r) .
$$

The steady-state generically exhibits a pitch-fork, period-doubling or NeimarkSacker bifurcation, if the first, second or third inequality in (B4) turns into an equality, respectively. Moreover, the dynamics is oscillating (i.e., the eigenvalues of the linearized system are complex) when

$$
\beta_{1}^{2}+4 \beta_{2}(1+r)<0 \text {. }
$$




\section{PROOF:}

Consider the steady-state $\left(p^{*}, n^{*}\right)$ with consistent forecasting rule, and notice that (B2) implies that either $p^{*}=p^{f}$ or $1-n^{*}=1+r$. The second case is impossible, so $p^{*}=p^{f}$ and, therefore, $n^{*}=0$.

Using (B2), the dynamics in deviations is given by

$$
p_{t}-p^{f}=\exp \left(-\left|p_{t-1}-p^{f}\right| / 200\right)\left(\frac{\beta_{1}}{1+r}\left(p_{t-1}-p^{f}\right)+\frac{\beta_{2}}{1+r}\left(p_{t-2}-p^{f}\right)\right) .
$$

The first term in the right hand-side is never greater than 1 . Thus, dynamics of (B5) is a superposition of contraction with linear process of second order

$$
(1+r) x_{t}=\beta_{1} x_{t-1}+\beta_{2} x_{t-2}
$$

with $x_{t}=p_{t}-p^{f}$. If the latter dynamics is locally stable, the steady-state $p^{f}$ of original dynamics (B5) will be also locally stable. Furthermore, since the exponential term in (B5) is equal to 1 in the steady-state, the linear parts of the dynamics of the last two processes are the same. Thus, processes (B5) and (B6) lose stability simultaneously and through the same bifurcation type.

The Jacobian matrix of (B6) in the steady-state is given by

$$
\boldsymbol{J}=\left\|\begin{array}{cc}
\frac{\beta_{1}}{1+r} & \frac{\beta_{2}}{1+r} \\
1 & 0
\end{array}\right\|
$$

The standard conditions for the stability can be expressed through the trace $\operatorname{Tr}(\boldsymbol{J})$ and the determinant $\operatorname{Det}(\boldsymbol{J})$ of this matrix, and are given by

$$
\operatorname{Tr}(\boldsymbol{J})<\operatorname{Det}(\boldsymbol{J})+1, \quad \operatorname{Tr}(\boldsymbol{J})>-1-\operatorname{Det}(\boldsymbol{J}), \quad \operatorname{Det}(\boldsymbol{J})<1 .
$$

Furthermore, the dynamics is oscillatory if $\operatorname{Tr}(\boldsymbol{J})^{2}-4 \operatorname{Det}(\boldsymbol{J})=0$. The substitution of the values of trace and determinant gives inequalities (B4) and condition $\beta_{1}^{2}+4 \beta_{2}\left(1+r_{f}\right)<0$ for the oscillations.

The bifurcation types can be determined from (B7), since when one of these inequalities turns to equality, the unit circle is crossed by a corresponding eigenvalue of the system. the second inequality is violated when an eigenvalue becomes equal to -1 , which implies the period-doubling bifurcation. The violation of the last inequality in (B7) implies that two complex eigenvalues cross the unit circle. This happens under the Neimark-Sacker bifurcation. Finally, consider the first inequality, which is violated when one eigenvalue becomes equal to 1 . It turns out that at this occasion two new steady-states are emerging, which implies that the system exhibits pitchfork bifurcation. Indeed, any steady-state $\left(p^{*}, n^{*}\right)$ should 


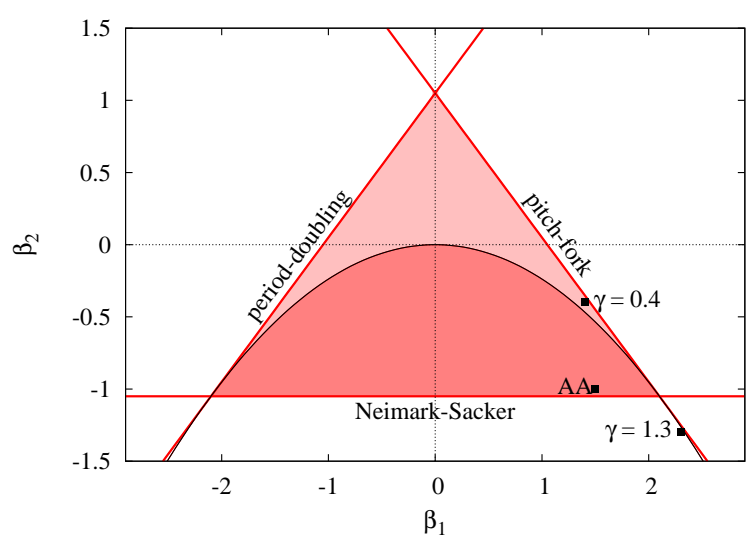

Figure B1. Stability of the fundamental Steady state under extrapolative expectations

Notes: The dynamics converge to the fundamental steady-state if the pair of coefficients $\left(\beta_{1}, \beta_{2}\right)$ of extrapolative rule belongs to the union of light and dark grey regions. The edges of the triangle at the border of the stability region correspond to pitchfork, period-doubling and Neimark-Sacker bifurcations. The price dynamics is oscillating if the pair $\left(\beta_{1}, \beta_{2}\right)$ lies below the parabolic curve. The three dots correspond to two trend-following heuristics (labeled $\gamma=0.4$ and $\gamma=1.3$ ) and an anchoring and adjustment heuristic (labeled AA), which are combined in the Heuristic Switching Model of Section 3 of the main text.

satisfy to

$$
\begin{aligned}
(1+r) p^{*} & =\left(1-n^{*}\right)\left(\left(1-\beta_{1}-\beta_{2}\right) p^{f}+\left(\beta_{1}+\beta_{2}\right) p^{*}\right)+n^{*} p^{f}+\bar{y} \\
(1+r) p^{*} & \stackrel{\Uparrow}{=} p^{f}+\left(\beta_{1}+\beta_{2}\right)\left(p^{*}-p^{f}\right)\left(1-n^{*}\right)+\bar{y} \\
& \stackrel{\mathbb{}}{=}\left(\beta_{1}+\beta_{2}\right)\left(p^{*}-p^{f}\right)\left(1-n^{*}\right)
\end{aligned}
$$

Thus, in any non-fundamental steady-state, the fractions of robots $n^{*}=1-$ $(1+r) /\left(\beta_{1}+\beta_{2}\right)$. Only if this fraction belongs to the interval $(0,1)$ two other steady-states exist with

$$
p_{ \pm}^{*}=p^{f} \pm 200 \log \left(1-n^{*}\right) .
$$

(The prediction rule is, of course, inconsistent in both steady-states.) When the first inequality in (B7) is satisfied, these two steady-state do not exist, but they appear at the moment when the inequality changes it sign.

In general, the dynamical system (B1) with homogeneous extrapolative expectations (B3) may have multiple steady-states. Proposition 2 asserts, however, that the extrapolative rule is consistent only in the fundamental steady state $p^{f}$. The stability conditions (B4) are illustrated in Fig. B1 in the parameter space $\left(\beta_{1}, \beta_{2}\right)$. The dark regions contain all rules for which the extrapolative heuristic 
(B3) generates stable dynamics. For the pairs lying below the parabolic curve, the dynamics are oscillating. A loss of (local) stability occurs when the pair $\left(\beta_{1}, \beta_{2}\right)$ leaves the stability area and crosses the boundary formed by the triangle. The dynamics immediately after the bifurcation are determined by the type of bifurcation through which stability is lost. For instance, after the pitchfork bifurcation the price diverges from its fundamental level and converges to one of two new stable steady-states. The Neimark-Sacker bifurcation implies existence of (quasi-)periodic price fluctuations right after the bifurcation.

The three dots shown in Fig. B1 correspond to three extrapolative forecasting rules estimated from individual experimental data. Two trend-following heuristics (Eq. (7) from the main text) with different values of the extrapolation coefficient $\gamma$ are labeled as $\gamma=0.4$ and $\gamma=1.3$. The anchoring and adjustment heuristic (Eq. (8) from the main text) is labeled as AA.

\section{Heterogeneity in HSTV05 Experiment}

HSTV05 estimated individual forecasting rules and found that many of them can be described by the extrapolative first order heuristics

$$
p_{t+1}^{e}=\alpha+\beta_{1} p_{t-1}+\beta_{2} p_{t-2} .
$$

Fig. C1 illustrates an extent of heterogeneity found in the HSTV05 experiment. For six different sessions of the Ro-HF treatment we show all individuals whose behavior was found to be consistent with the extrapolative heuristic. ${ }^{1}$ The dots represent the coefficients of the estimated heuristics, with coefficient $\beta_{1}$ shown on the horizontal axis and coefficient $\beta_{2}$ on the vertical axis. Different regions of the space correspond to different qualitative dynamics of the model with corresponding homogeneous expectation rule as derived in Appendix B. The dynamics converge to the fundamental steady-state if the pair of coefficients $\left(\beta_{1}, \beta_{2}\right)$ belongs to the union of light and dark grey regions and diverge otherwise. The price dynamics is oscillating if the pair $\left(\beta_{1}, \beta_{2}\right)$ lies below the parabolic curve.

While the dispersion of individual forecasting rules is clear, the figure suggests some regularities. In the converging sessions 2 and 5 , the majority of rules belongs to the region of monotonic convergence. In contrast, in the oscillating sessions almost all individual rules lie in the oscillatory region (i.e., the linear forecasting rule has complex eigenvalues). Furthermore, in sessions 1 and 6 with constant price oscillations at least two individual rules in every group are very close to the stability border of the Neimark-Sacker bifurcation (i.e., are close to complex unit roots), while in groups 4 and 7 with dampened oscillations both stable and unstable rules were present.

\footnotetext{
${ }^{1}$ In every group there were rules which cannot be represented by the extrapolative prediction, e.g., an adaptive heuristic or linear rules with three lags.
} 

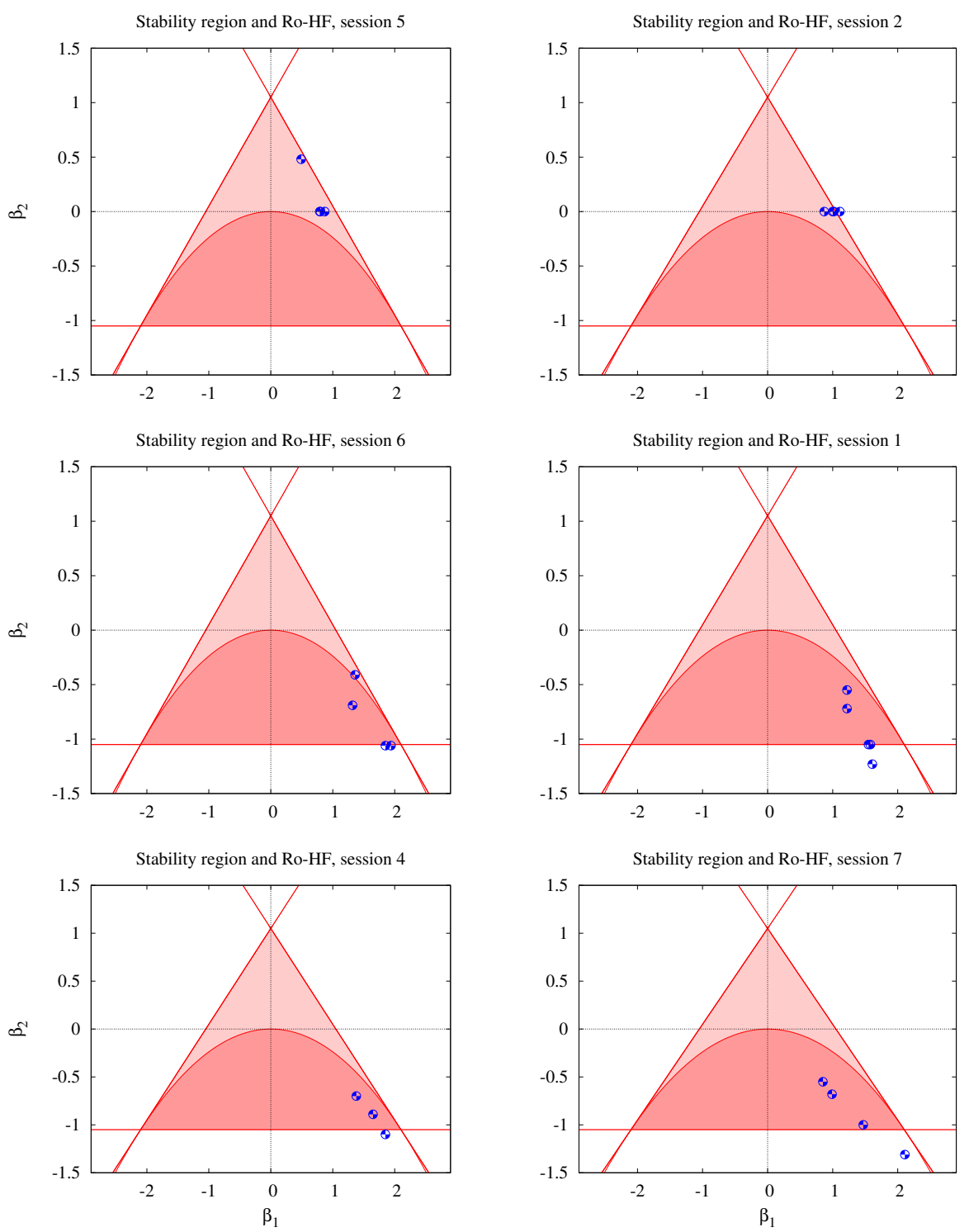

Figure C1. Stability of homogeneous expectations model with eXtrapolative Rules estimated IN THE EXPERIMENT 


\section{EMPIRICAL VALIDATION}

\section{D1. One-period ahead simulations}

The plots of this section illustrate the one-period ahead forecast by the HSM for different groups of the learning to forecast asset pricing experiment. The left panels of the plots show the prices in experiment (line with points) with corresponding one-step ahead predictions of the HSM (points). The right panels show the corresponding evolution of fractions of the four heuristics: adaptive expectations (ADA), weak trend followers (WTR), strong trend followers (STR) and learning anchoring and adjustment heuristic (LAA).

Fig. D1 (which complements Fig. 6 of the main text) shows the fit for three groups of the HSTV05 experiment with "robots" and high fundamental price, $p^{f}=60$. These groups illustrate three qualitative features of aggregate data observed in this experiment: almost monotonic convergence (group 2), oscillations with constant amplitude (group 1) and damping oscillations (group 7). These are the groups for which Anufriev and Hommes (2012) made a 50-periods ahead simulations.

Notice, that the domination of the LAA rule happens much faster in simulations for group 1, than for group 6. (For instance, $80 \%$ impact is reached by the LAA rule after 20 periods for group 1 and after 40 periods for group 6.) This difference reflects the fact that the frequency of oscillations in the two experimental groups were not the same. During the experiment we observe about 6 "cycles" in group 1 , but only about 4 and a half "cycles" in group 6 .

Two panels of Fig. D2 give the examples with a low fundamental price of 40, whose strong oscillations are captured by an initially dominating STR rule, until the LAA rule becomes dominating after period 40. This figure complements the top panels of Fig. 8 of the main text.

Fig. D3 is an example of the environment without robot traders. (It complements the middle panels of Fig. 8 of the main text.) Large amplitude price oscillations, with prices almost reaching their maximum 100 and minimum 0 , arise, but prices stabilize towards the end of the experiment. The evolution of the impacts of the four forecasting heuristics is similar to the case of dampening price oscillations, with the strong trend rule (STR) initially dominating the market, followed by the anchor and adjustment rule (LAA) taking over around period 25, and the adaptive expectations (AA) rule finally dominating towards the end.

Finally, Figs. D4 and D5 in the "bubble experiments" of the HSTV08 is another example without robot traders, this time with a much larger upper-bound of 1000 (instead of 100). (See also the bottom panel of Fig. 8 of the main text.) The asset price oscillates with very large amplitude, with a long lasting rend of 25 periods with prices rising close to their maximum 1000, after which a crash follows to values close to their minimum 0 , and the market starts oscillating. The evolution of the impacts of the four forecasting heuristics is similar as before, with the STR 

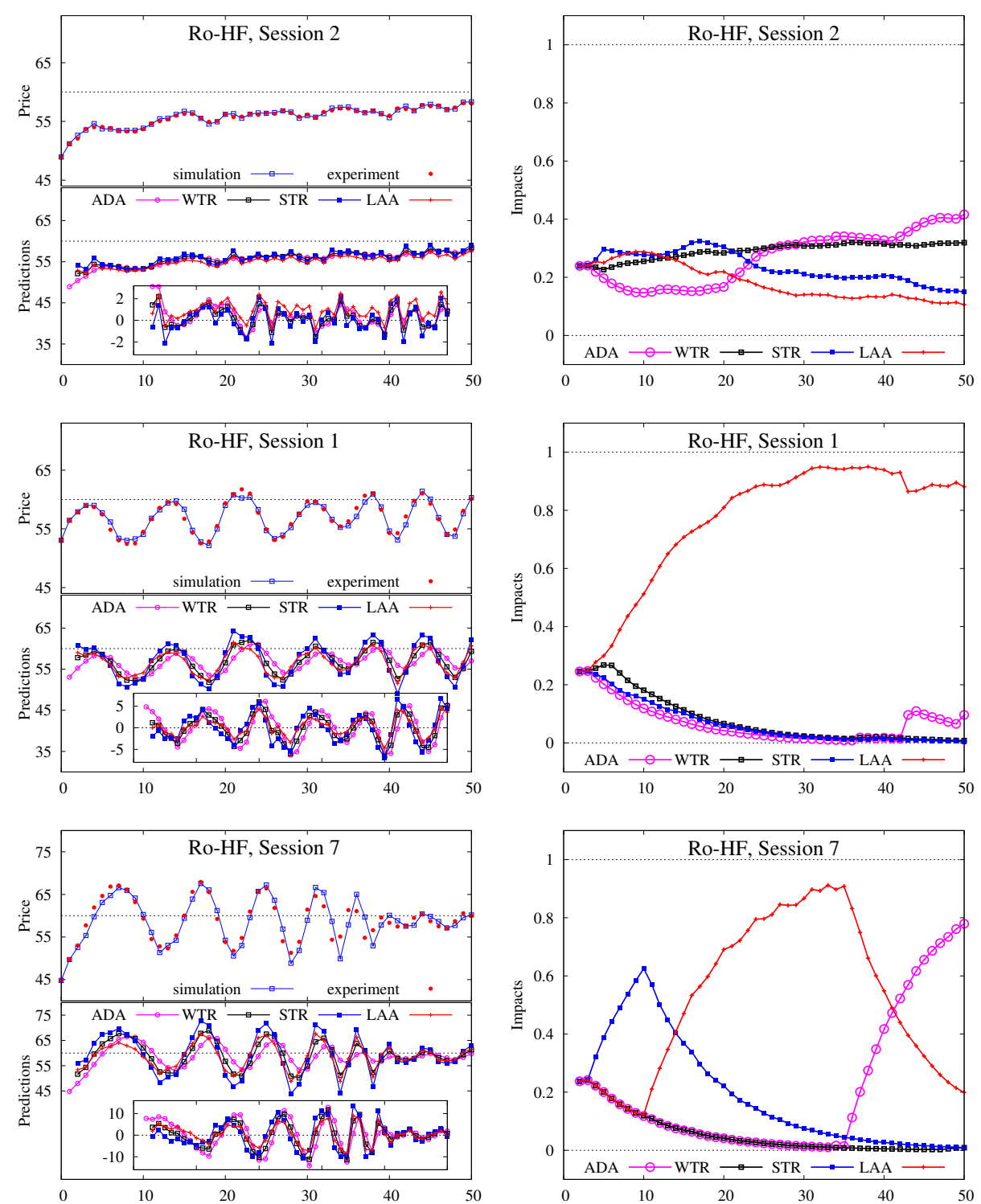

Figure D1. LABoratory EXPERIMENTS AND the HSM IN 3 SESSIONS From Ro-HF HSTV05 EXPERIMENT

Notes: This Figure compements Fig. 6 of the main text. Three sessions with different qualitative dynamics are shown. The upper parts of left panels show prices for experiments with corresponding one-step ahead predictions of the HSM. The lower parts show predictions and forecasting errors (inner frames) of the four heuristics. The right panels show the evolution of the impacts of the four heuristics. 

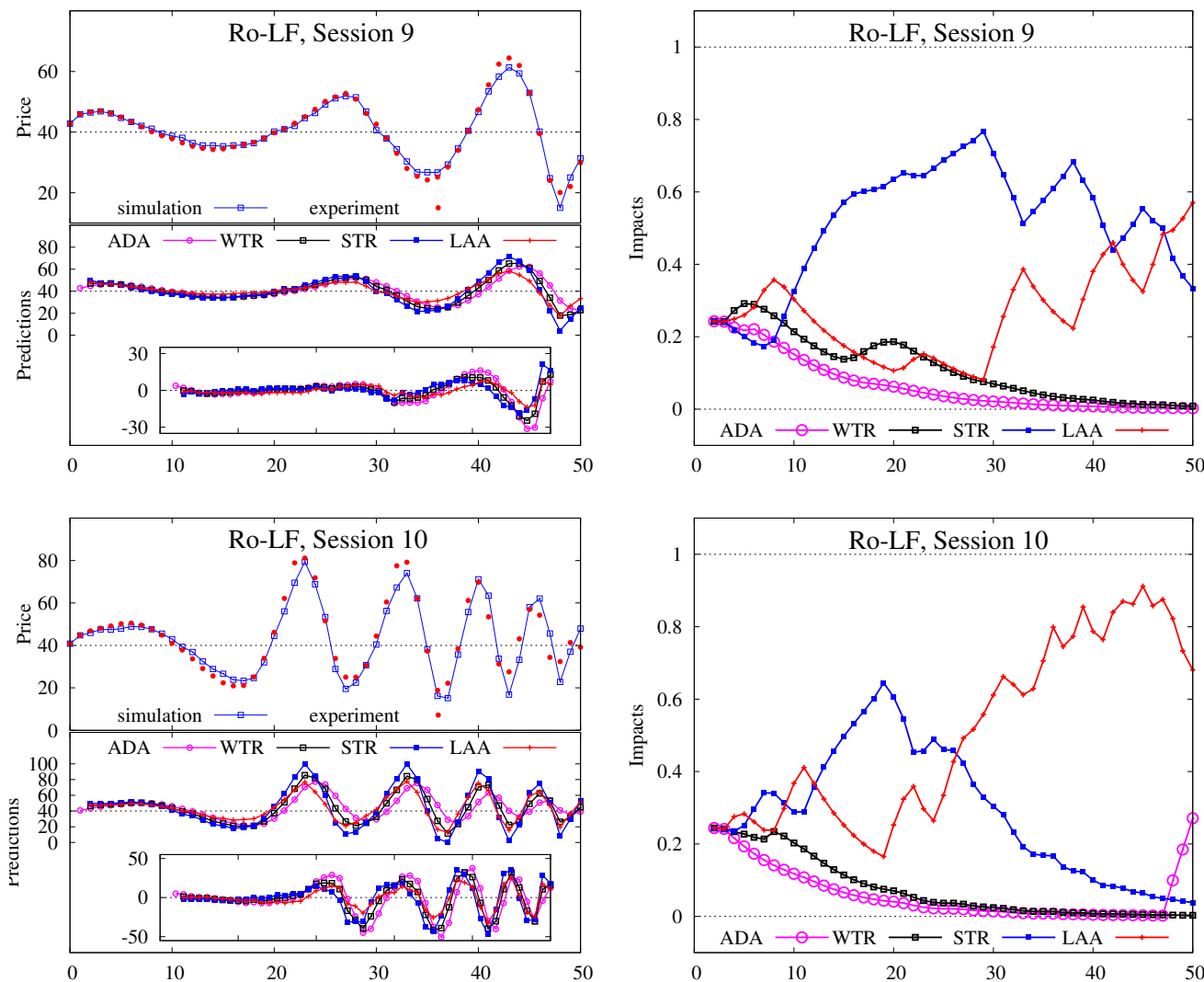

Figure D2. Laboratory experiments and the HSM in 2 SESSiOnS From Ro-LF HSTV05 EXPeriment

Notes: This Figure compements the top panels of Fig. 8 of the main text. The upper parts of left panels show prices for experiments with corresponding one-step ahead predictions of the HSM. The lower parts show predictions and forecasting errors (inner frames) of the four heuristics. The right panels show the evolution of the impacts of the four heuristics.

rule taking the lead, increasing its share to more than $80 \%$ after 25 periods, then slowly declining and finally dominated by the LAA rule with more than $60 \%$ of the share after 50 periods.

We summarize the corresponding evolution of heuristics' impacts in Table D1. 

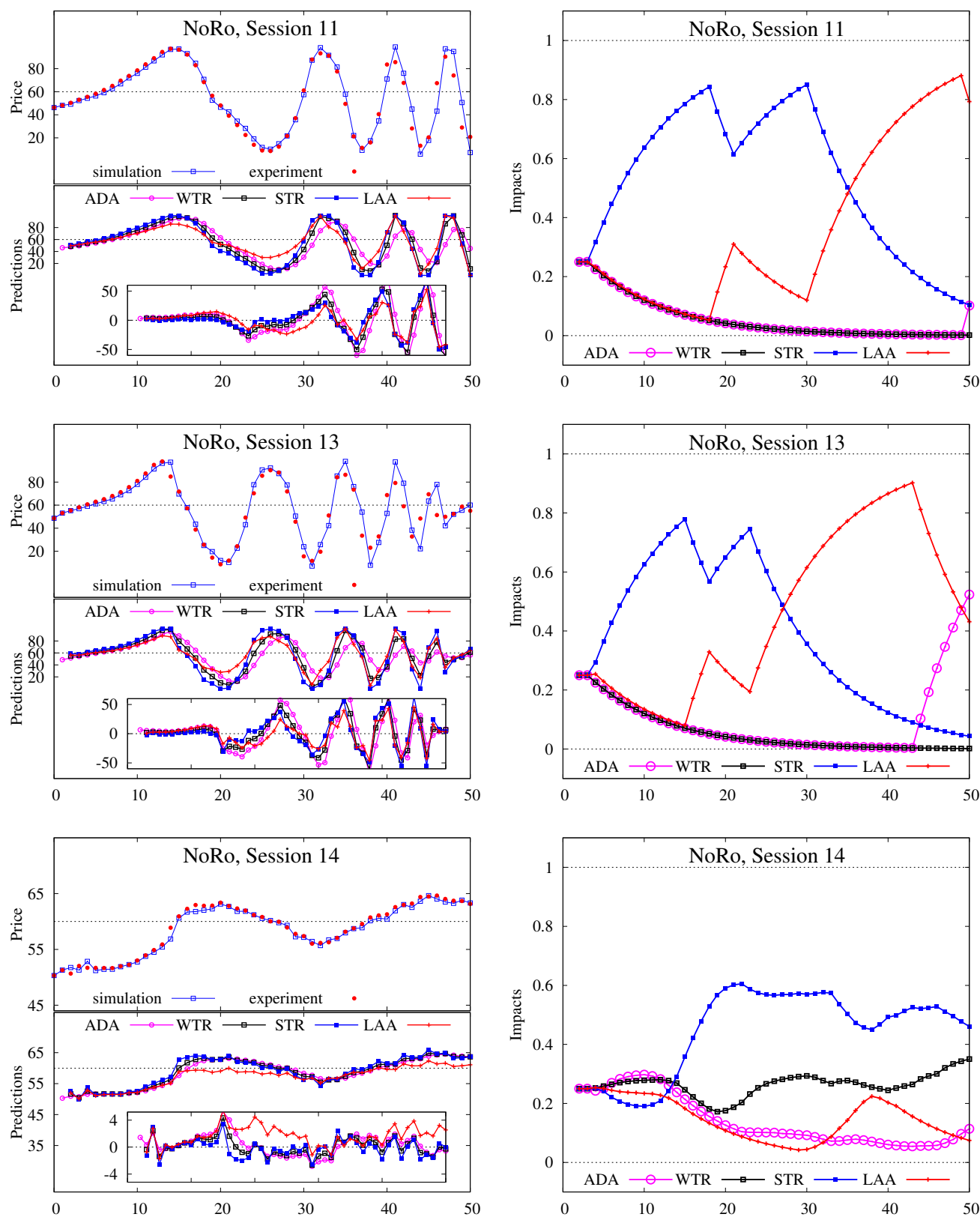

Figure D3. LABORATORY EXPERIMENTS AND THE HSM IN 3 SESSIONS FrOM NoRo HSTV05 EXPERIMENT

Notes: This Figure compements the middle panels of Fig. 8 of the main text. The upper parts of left panels show prices for experiments with corresponding one-step ahead predictions of the HSM. The lower parts show predictions and forecasting errors (inner frames) of the four heuristics. The right panels show the evolution of the impacts of the four heuristics. 

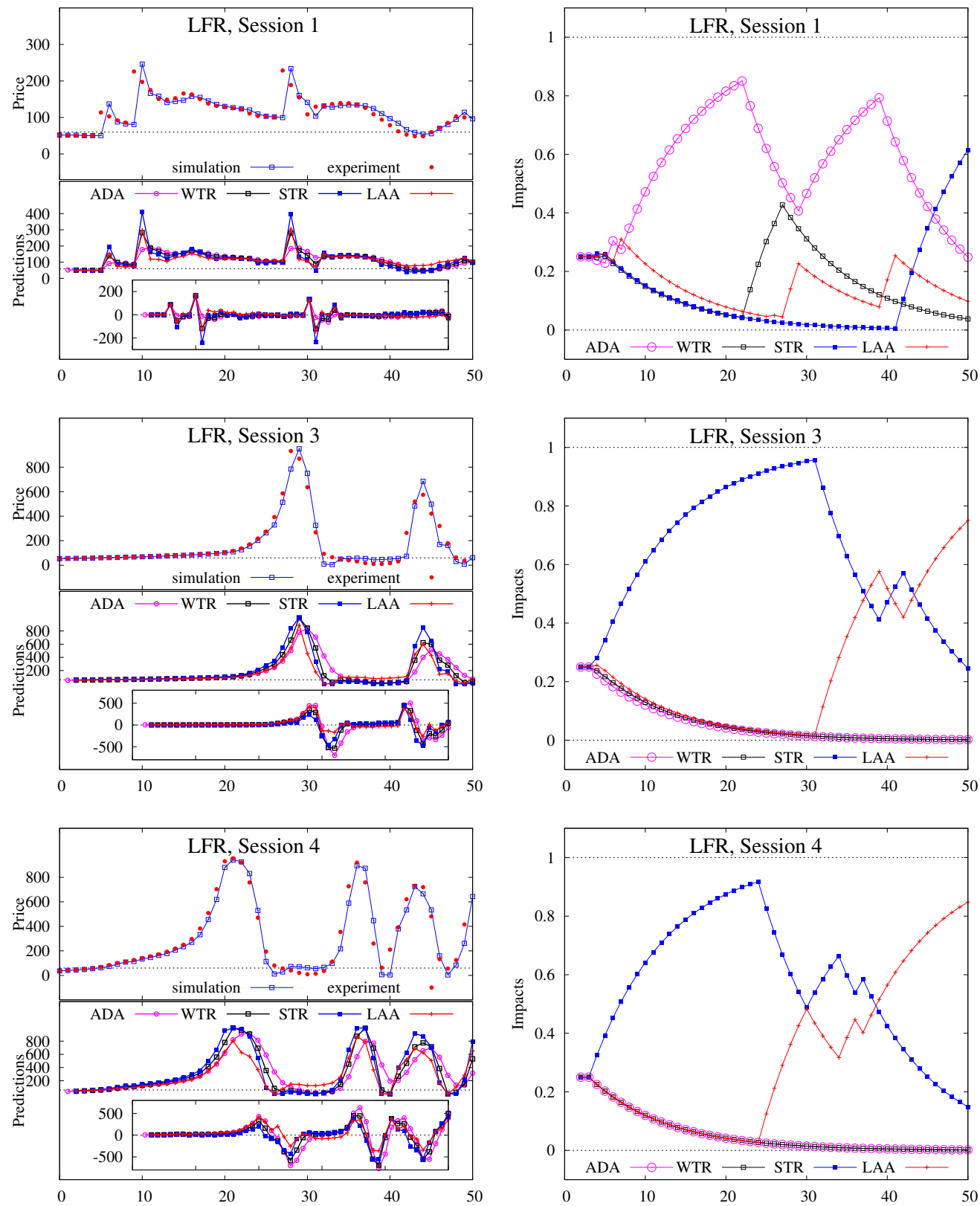

Figure D4. Laboratory eXPERIMENTS AND the HSM in 3 SESSiOns From LFR HSTV08 EXPERIMENT.

Notes: This Figure compements the bottom panels of Fig. 8 of the main text. The upper parts of left panels show prices for experiments with corresponding one-step ahead predictions of the HSM. The lower parts show predictions and forecasting errors (inner frames) of the four heuristics. The right panels show the evolution of the impacts of the four heuristics. 

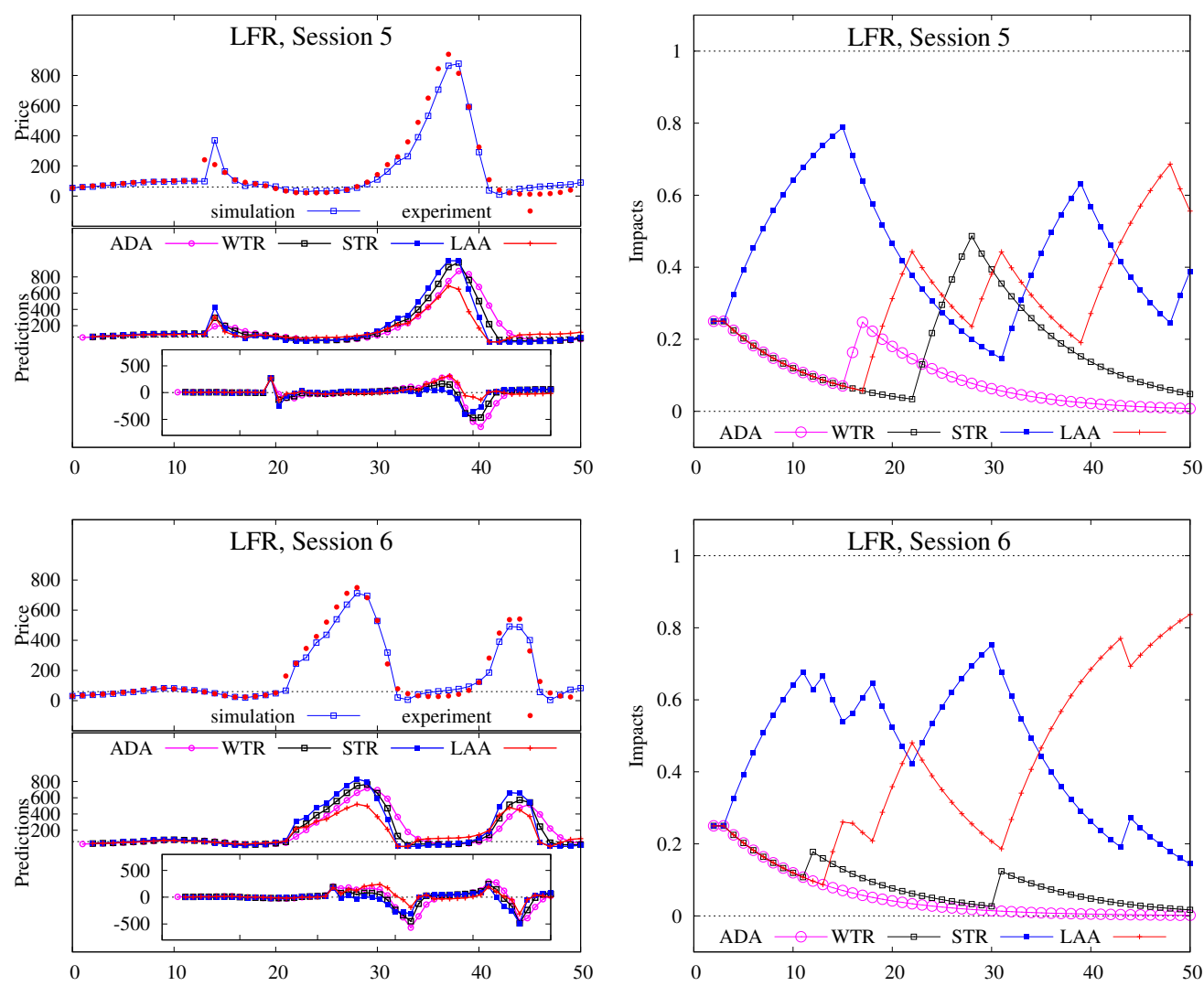

Figure D5. LABORATORY EXPERIMENTS AND THE HSM IN 3 SESSIONS FROM LFR HSTV08 EXPERIMENT.

Notes: This Figure compements the bottom panels of Fig. 8 of the main text. The upper parts of left panels show prices for experiments with corresponding one-step ahead predictions of the HSM. The lower parts show predictions and forecasting errors (inner frames) of the four heuristics. The right panels show the evolution of the impacts of the four heuristics. 
Table D1-Heuristic Switching Model in the Learning to Forecast Experiments

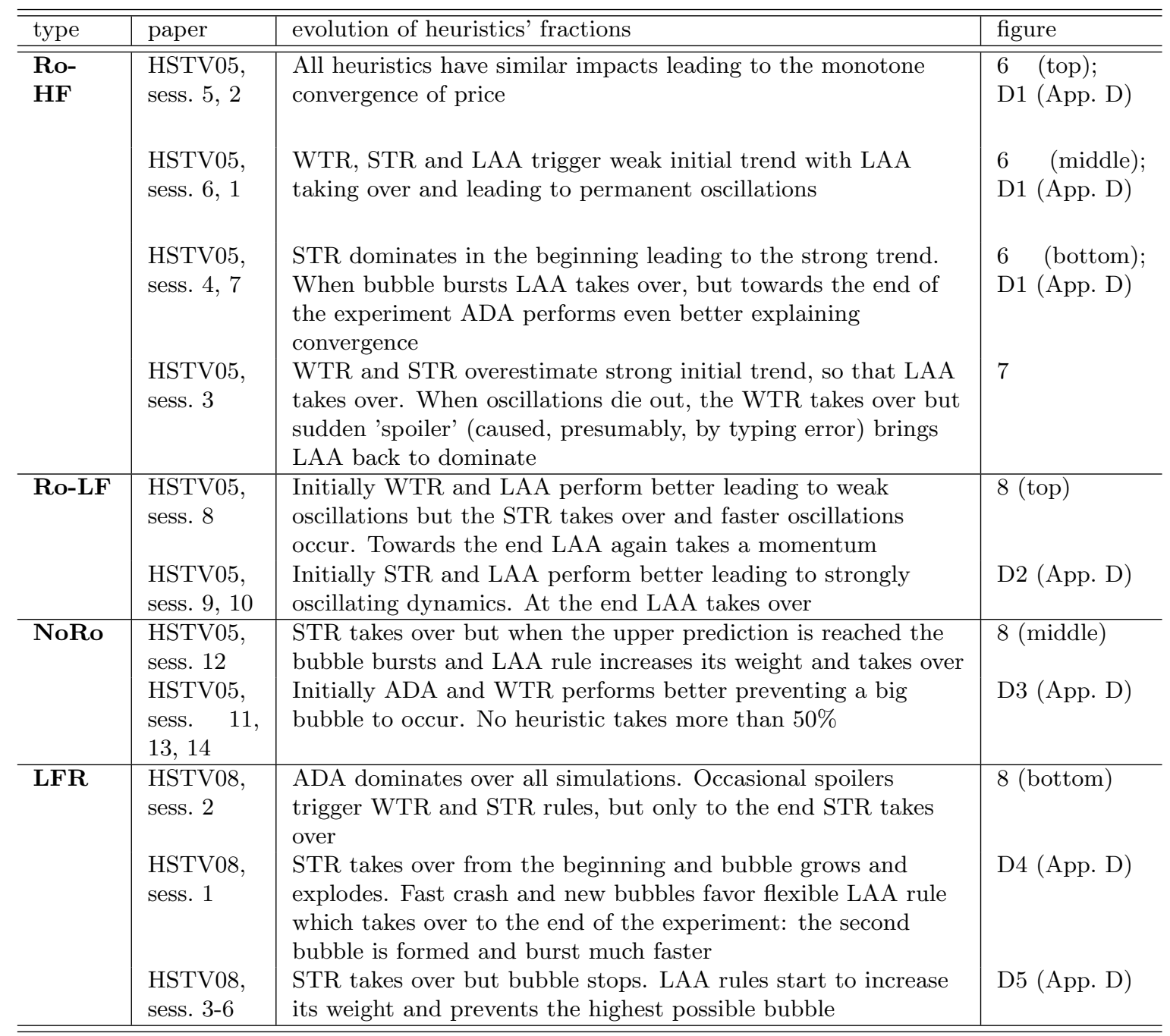




\section{D2. Forecasting performance}

The tables of this section supplement the results of Sections 4.2 and 4.3 of the main text. We investigate the forecasting performance of the HSM with benchmark learning parameters $\beta=0.4, \eta=0.7$ and $\delta=0.9$, and compare it with the performance of other models.

Table D2 (which complements Table 2 of the main text) shows the mean squared prediction errors of the one-step ahead forecast for different homogeneous and heterogeneous expectation models. The best model has smallest MSE. The best among four homogeneous model corresponding to four different heuristics is shown in bold. Also in bold we show the MSE for the HSM with benchmark parameters. The best among all the model is shown in italic, and it is often the HSM where learning parameters are chosen to minimize the MSE.

Tables D3 and D4 complement Table 3 of the main text. They show the outof-sample forecasting errors from 1- to 7-periods ahead of three different model: the HSM optimizied in-sample, the HSM with the benchmark parameters and the $\operatorname{AR}(2)$ model optimized in-sample.

Finally, Table D5 (which complements Table 4 of the main text) shows the 1and 2-periods ahead forecasting errors averaged over moving window for the same three models. The smallest forecasting errors within the same class are shown in italic. 
VOL. VOLUME NO. ISSUE INDIVIDUAL EXPECTATIONS AND AGGREGATE OUTCOMES 19

TABle D2-MSE of the ONE-STEP AHEAd Forecast For 7 SEssions of HSTV05 ANd 5 Session of HSTV08

\begin{tabular}{lcccccccc}
\hline \hline & Ro-HF & \multicolumn{2}{c}{ Ro-LF } & \multicolumn{1}{c}{ NoRo } \\
Specification & $\mathbf{2}$ & $\mathbf{1}$ & $\mathbf{7}$ & $\mathbf{9}$ & $\mathbf{1 0}$ & $\mathbf{1 1}$ & $\mathbf{1 3}$ & $\mathbf{1 4}$ \\
\hline Fundamental prediction & 16.62 & 15.76 & 21.91 & 108.91 & 309.62 & 877.18 & 631.89 & 16.65 \\
Naive heuristic & 0.04 & 3.54 & 13.25 & 20.05 & 122.50 & 297.92 & 251.31 & 0.53 \\
\hline ADA heuristic & $\mathbf{0 . 0 7}$ & 5.67 & 19.52 & 38.98 & 197.59 & 452.77 & 376.74 & 1.20 \\
WTR heuristic & 0.09 & 2.09 & 9.29 & 9.20 & 72.28 & 200.62 & 180.08 & $\mathbf{0 . 2 5}$ \\
STR heuristic & 0.50 & 2.91 & 14.72 & $\mathbf{4 . 1 0}$ & 80.67 & $\mathbf{1 1 2 . 6 5}$ & 209.37 & 0.41 \\
LAA heuristic & 0.46 & $\mathbf{0 . 4 6}$ & $\mathbf{5 . 8 6}$ & 16.47 & $\mathbf{4 8 . 1 1}$ & 150.73 & $\mathbf{1 3 8 . 2 9}$ & 4.77 \\
\hline 4 heuristics $(\delta=1)$ & 0.08 & 1.24 & 7.10 & 7.43 & 52.20 & 156.83 & 135.91 & 0.65 \\
4 heuristics (Figs.) & $\mathbf{0 . 0 6}$ & $\mathbf{0 . 4 7}$ & $\mathbf{4 . 3 2}$ & $\mathbf{2 . 4 4}$ & $\mathbf{2 6 . 5 8}$ & $\mathbf{7 0 . 2 5}$ & $\mathbf{8 2 . 7 3}$ & $\mathbf{0 . 3 3}$ \\
4 heuristics (best fit) & 0.05 & 0.44 & 2.92 & 1.52 & 23.82 & 68.43 & 72.39 & 0.21 \\
\hline$\beta \in[0,10]$ & 10.00 & 0.10 & 0.23 & 0.07 & 0.20 & 1.84 & 0.54 & 0.07 \\
$\eta \in[0,1]$ & 0.42 & 1.00 & 0.45 & 0.96 & 0.74 & 0.72 & 0.70 & 0.95 \\
$\delta \in[0,1]$ & 0.89 & 0.45 & 0.46 & 0.79 & 0.77 & 0.84 & 0.00 & 0.06 \\
\hline
\end{tabular}

\begin{tabular}{lccccc}
\hline \hline & \multicolumn{5}{c}{$\mathbf{L F R}^{a}$} \\
Specification & $\mathbf{1}$ & $\mathbf{3}$ & $\mathbf{4}$ & $\mathbf{5}$ & $\mathbf{6}$ \\
\hline Fundamental prediction & 5577.12 & 65601.01 & 159202.38 & 67411.56 & 67085.61 \\
Naive heuristic & 1057.02 & 12452.97 & 26736.05 & 6905.15 & 7634.30 \\
\hline ADA heuristic & $\mathbf{1 0 7 5 . 9 3}$ & 20362.17 & 42040.69 & 12868.66 & 14028.82 \\
WTR heuristic & 1284.48 & 7652.45 & 16912.16 & 3684.20 & 4102.32 \\
STR heuristic & 2958.17 & $\mathbf{4 8 0 4 . 1 0}$ & $\mathbf{7 8 4 4 . 2 7}$ & $\mathbf{2 1 9 8 . 9 1}$ & $\mathbf{2 4 0 6 . 2 1}$ \\
LAA heuristic & 1837.39 & 9183.66 & 15038.59 & 10763.49 & 9028.54 \\
\hline 4 heuristics $(\delta=1)$ & 1376.33 & 5931.38 & 12086.66 & 3399.96 & 3408.68 \\
4 heuristics (Figs.) & $\mathbf{1 1 3 3 . 8 9}$ & $\mathbf{3 3 1 2 . 3 8}$ & $\mathbf{4 8 4 6 . 4 4}$ & $\mathbf{2 8 8 7 . 8 4}$ & $\mathbf{1 8 3 2 . 6 5}$ \\
4 heuristics (best fit) & 1033.03 & 3075.22 & 4795.97 & 2203.65 & 1421.88 \\
\hline$\beta \in[0,10]$ & 0.20 & 10.00 & 0.51 & 0.07 & 5.93 \\
$\eta \in[0,1]$ & 0.80 & 1.00 & 0.70 & 0.39 & 0.87 \\
$\delta \in[0,1]$ & 0.70 & 0.00 & 0.88 & 0.95 & 0.92 \\
\hline
\end{tabular}

Notes: 9 different model specifications are compared.

${ }^{a}$ For the LFR HSTV08 experiment MSE is computed over 46 periods. 


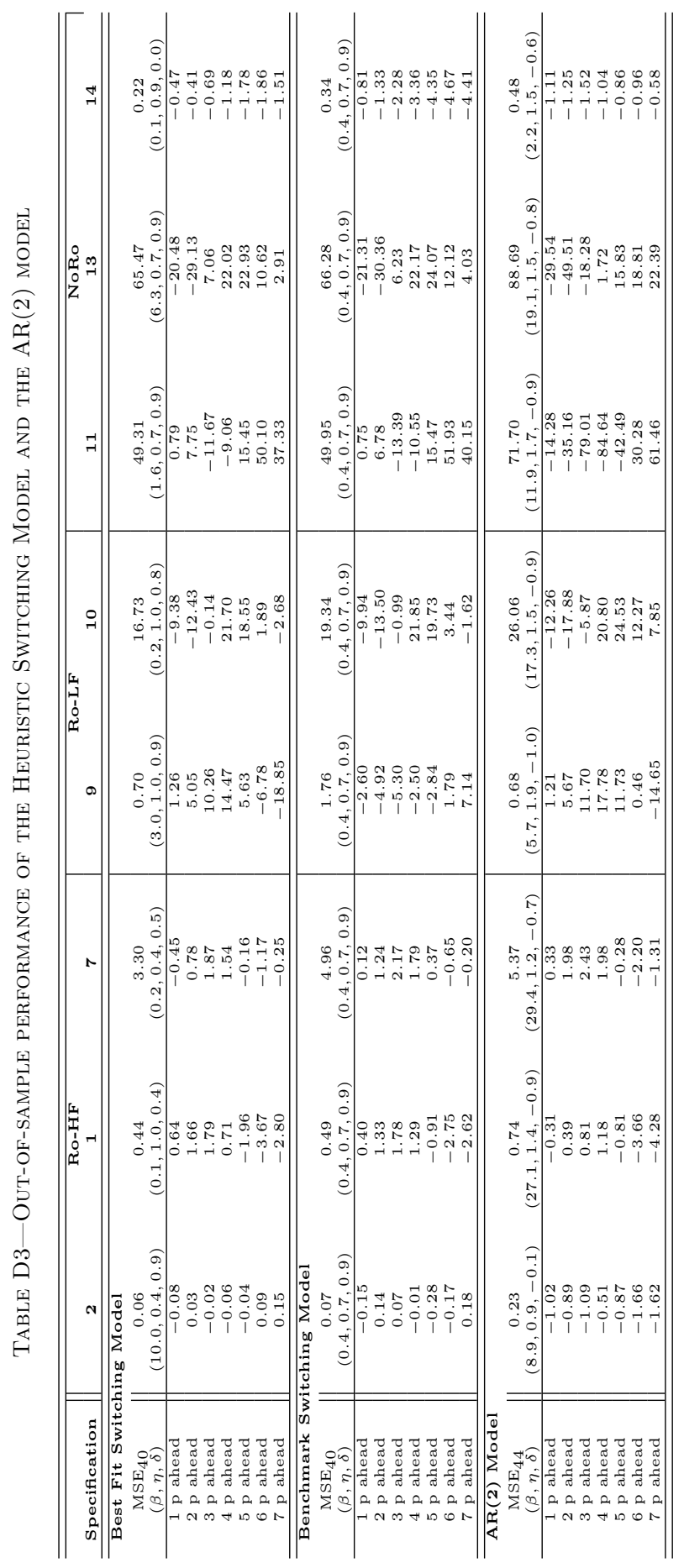


VOL. VOLUME NO. ISSUE INDIVIDUAL EXPECTATIONS AND AGGREGATE OUTCOMES 21

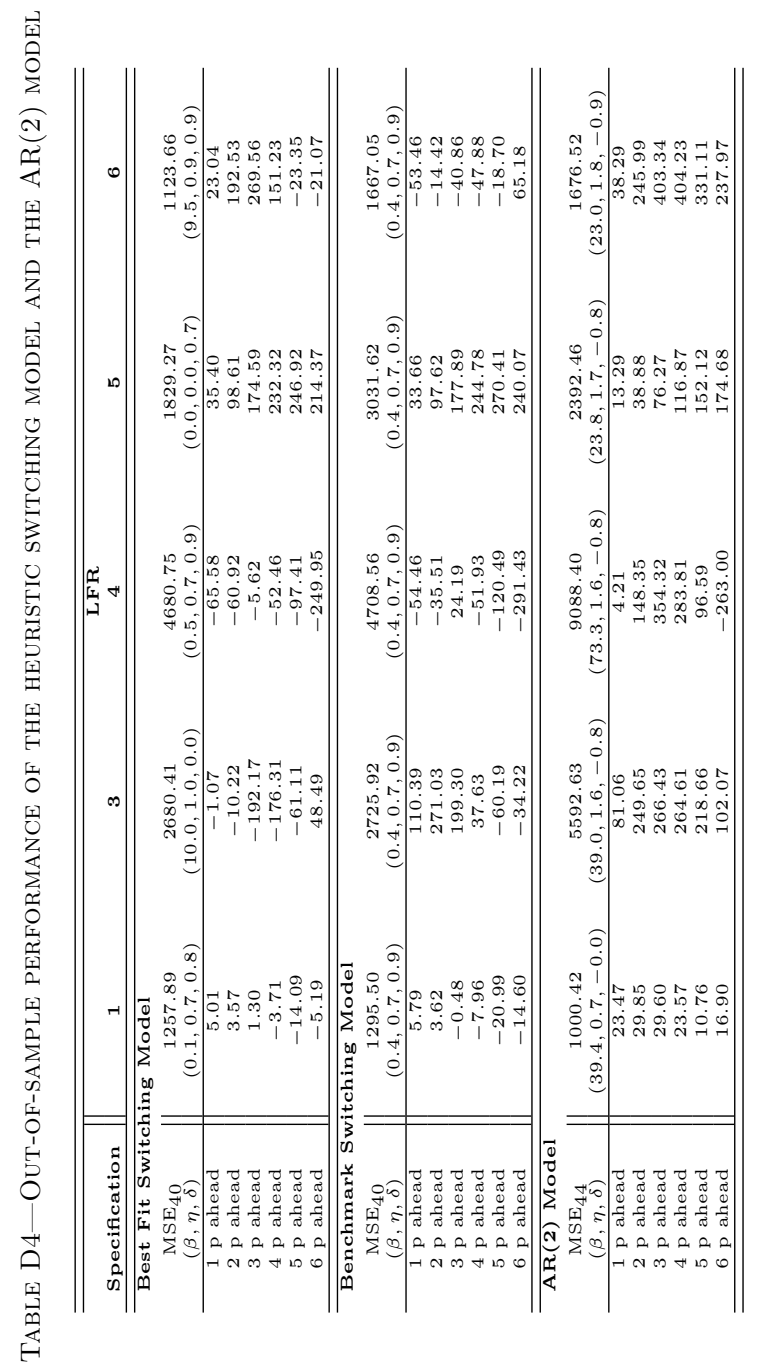




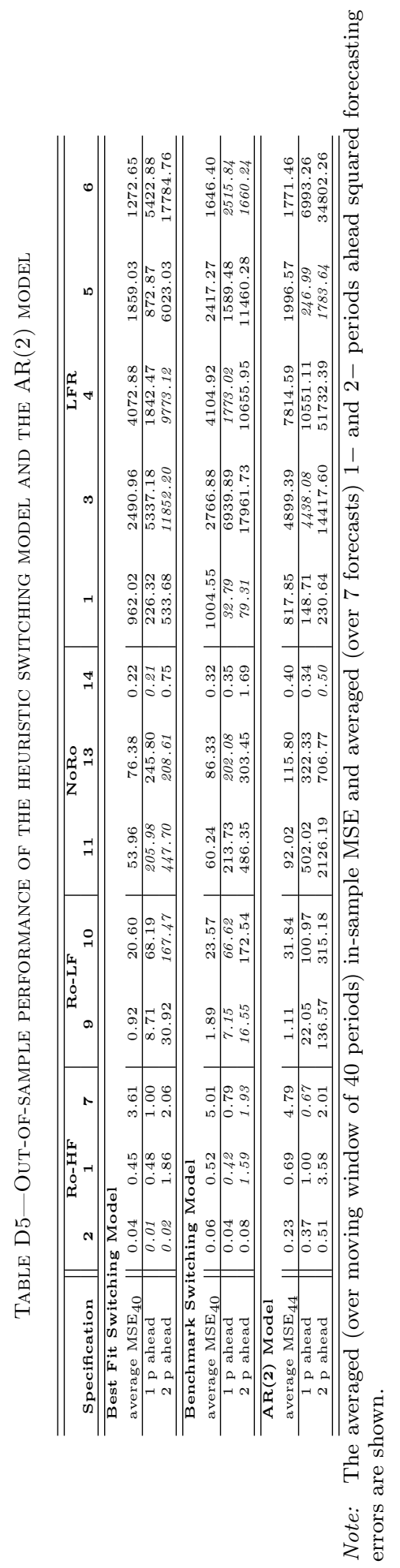

